\title{
Determination of azithromycin heteroresistant Campylobacter jejuni in traveler's diarrhea
}

\author{
Woradee Lurchachaiwong ${ }^{1 *}$, Supaporn Ruksasiri ${ }^{1}$, Patcharawalai Wassanarungroj ${ }^{1}$, Oralak Serichantalergs ${ }^{1}$, \\ Ladaporn Bodhidatta', John Crawford' ${ }^{1}$ Sanjaya Kumar Shrestha ${ }^{2}$ and Prativa Pandey ${ }^{3}$
}

\begin{abstract}
Campylobacter is the most common cause of traveler's diarrhea (TD) and human bacterial gastroenteritis. A heteroresistant Campylobacter jejuni (C. jejuni) isolate, identified by microbiological methods and characterized with molecular techniques, was obtained from a traveler in Nepal suffering TD. The presence of atypical colonies within the clear zone of inhibition was the first evidence of an atypical phenotype, leading to additional characterization of this heteroresistant strain. Antimicrobial susceptibility testing (AST) and population analysis profiling (PAP) demonstrated heteroresistance to azithromycin (AZM), a first-line antibiotic treatment for Campylobacter infections. Molecular analysis indicated a point mutation occurred on the $23 \mathrm{~S}$ rRNA gene at the A2075G transitions, and the number of mutated gene copies was proportional to AZM resistance. Heteroresistant C. jejuni subpopulations from acute TD are likely underestimated, which may lead to treatment failures, as was the case for this patient. The presence of a heteroresistant strain in a high antibiotic environment may select for additional drug resistance and enable distribution into hospital and local communities.
\end{abstract}

Keywords: Heteroresistance, Campylobacter jejuni, Azithromycin, Travelers

\section{Background}

Campylobacter is a Gram-negative bacteria causing travelers' diarrhea (TD) in US military personnel during deployment to developing countries [1], as well as a leading cause of TD in Nepal and bacterial foodborne illness worldwide $[2,3]$. The vast majority of these intestinal infections are caused by Campylobacter jejuni (C. jejuni) and Campylobacter coli (C. coli), of which approximately $90 \%$ are attributed to $C$. jejuni [3]. Macrolides, primarily azithromycin (AZM) or erythromycin (ERY), remain the frontline agents for treating Campylobacter infections [4]; however, a progressive increase in Campylobacter macrolide resistance was reported in some countries and is a growing health threat concern

\footnotetext{
*Correspondence: woradeel.fsn@afrims.org

1 Department of Bacterial and Parasitic Diseases, Armed Forces Research Institute of Medical Sciences, 315/6 Rajvithi Road, Bangkok 10400,

Thailand

Full list of author information is available at the end of the article
}

[5]. The term "heteroresistance" describes a phenomenon where subpopulations of seemingly isogenic bacteria exhibit a range of susceptibilities to a particular antibiotic [6]. The mechanism of action with macrolides resistance was traced to a mutation on domain $\mathrm{V}$ of the $23 \mathrm{~S}$ rRNA. Single base substitution positions, as well as the number of mutated $23 \mathrm{~S}$ rRNA copies, are correlated to macrolide resistance and proportion levels of minimum inhibitory concentrations (MICs) [7]. As a result of genotypic variation, the Clinical and Laboratory Standards Institute (CLSI) developed clinical laboratory standards and recommendations regarding antimicrobial resistance [6]. Until recently, heteroresistant patterns were poorly understood and the application of consensusbased standards for this phenotype was controversial. Confusion on this phenomenon precludes establishing its clinical significance, leading to improper therapeutic interventions and ambiguous guidelines. Not recognizing AZM heteroresistance in $C$. jejuni may lead to treatment failure, and as such lead to increasing resistance. Hence, 
this study characterized AZM heteroresistant C. jejuni from acute diarrheal illness in travelers to Nepal. The early detection of heteroresistant Campylobacter isolates enables medical providers to make appropriate treatment decisions.

\section{Methods}

A total of 105 Campylobacter isolates, including 88 C. jejuni and 17 C. coli were obtained from 105 travelers with acute diarrheal illness seeking healthcare at the CIWEC Clinic Travel Medicine Center in Kathmandu, Nepal during 2012-2014. Antimicrobial susceptibility testing (AST) to azithromycin (AZM), erythromycin (ERY), nalidixic acid (NAL), and ciprofloxacin (CIP) was performed on confirmed isolates using a commercially available E-test method (Liofilchem, Roseto degli Abruzzi TE, Italy) to determine the minimal inhibitory concentration (MIC). The AST results were interpreted following CLSI guidelines and National Antimicrobial Resistance Monitoring System (NARMS) using Campylobacter jejuni ATCC 33560 as the control strain [8, 9]. AST observations of the heterogeneous phenotype was the presence of discrete colonies in the clear zone of inhibition. These isolates were recorded as "suspected heteroresistance" and the discrete colonies were harvested for heteroresistant determinations. The colonies showing suspected heteroresistance were submitted for capsule genotyping by multiplex PCR [10]. The colonies from the same specimens that have the same capsule genotypes, but discriminate AST profiles, were subsequently assessed with the "population analysis profiling (PAP)" which remains the gold standard for detecting heteroresistance. Isolates were determined to be heteroresistant when the lowest antibiotic concentration giving the maximum growth inhibition was eightfold higher than the highest non-inhibitory concentration $[6,11,12]$. Furthermore, these isolates were sequenced at the domain $\mathrm{V}$ of the 23S rRNA gene, as described elsewhere [7]. Briefly, genomic DNA extraction for C. jejuni and C. coli was be prepared using the DNeasy Blood \& Tissue kit (Qiagen Valencia, CA) according to manufacturer's directions. Primers flanking each operon were utilized to amplify the three copies of the 23S rRNA gene in C. coli and C. jejuni isolates. Amplified products were analyzed by gel electrophoresis and ethidium bromide staining was used to determine the amplicon sizes. The amplified products were further purified using the Wizard PCR purification system (Promega, Madison, WI) and submitted for sequencing using primers specific for the $23 \mathrm{~S}$ domain $\mathrm{V}$. The $508 \mathrm{bp}$ of $23 \mathrm{~S}$ rRNA sequences were analyzed to identify potential macrolide mutations in each copy target gene by Sequencher version 5.0 DNA sequence analysis software (Gene Codes Corporation, Ann Arbor, MI).

\section{Results and discussion}

Of 105 Campylobacter isolates, one $C$. jejuni was found to be heteroresistant to AZM. This acute diarrheal stool sample came from a traveler who presented to the clinic with fever, abdominal pain, nausea, vomiting, diarrhea and fatigue. The stool characteristic was watery without mucus and blood. The sample was negative for enteric viruses, gastrointestinal parasites, and enteric bacterial pathogens including diarrheagenic E. coli; however, colonies identified as $C$. jejuni were detected.

The AST profile of this heterogeneous C. jejuni isolate was MICs $\geq 256 \mu \mathrm{g} / \mathrm{ml}$ to CIP, ERY, and NAL, and presenting resistance to CIP, ERY, and NAL, respectively. This Campylobacter isolate displayed a heterogeneous phenotype by presenting as speckle colonies in the clear zone of inhibition to AZM. Repetitive AST testing to AZM of the original Campylobacter isolate, as well as colonies from the outer and the inner (speckle colonies) inhibition zone, were confirmed by E-test to make the heteroresistant determination. The results were reproducible, presenting with a $\mathrm{MIC}=0.19 \mu \mathrm{g} / \mathrm{ml}$; however, the speckle colonies on the inner inhibition zone showed inconsistent MIC result to AZM (Fig. 1). Subsequently, the original Campylobacter isolate, colonies from outer inhibition zone, and three random speckle colonies from the inner inhibition zone were submitted for capsule genotyping by multiplex PCR. All isolates possessed the same capsule type to HS2, which potentially has a common Penner type of $C$. jejuni isolated from South and Southeast Asia [10], as well as to a previous report of fluoroquinolone resistance in Hawaii [13].

Regarding the PAP result, the laboratory resistant and heteroresistant isolates exhibited different growth patterns between the lowest concentrations to maximum inhibition and the highest non-inhibitory concentration when compare to the ATCC 33560 C. jejuni control

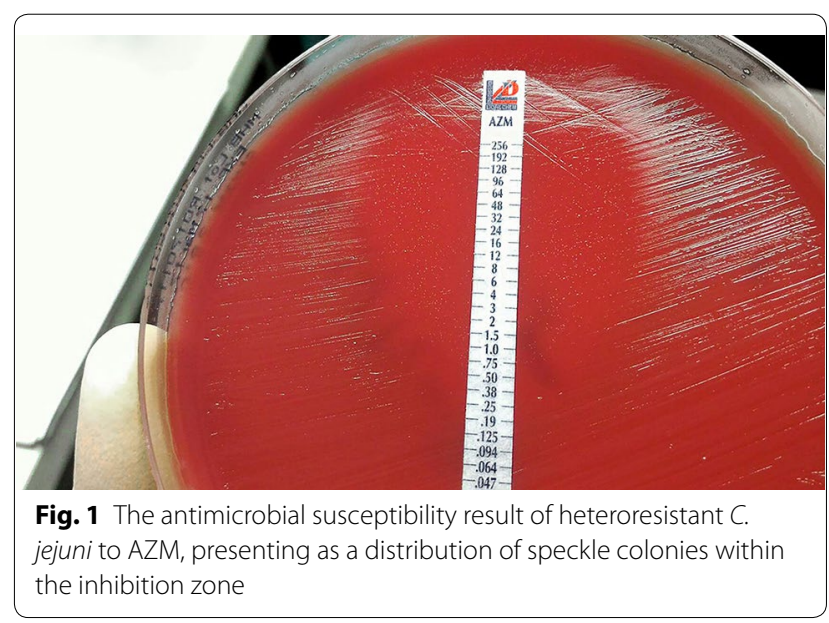


strain (Fig. 2). The laboratory isolate demonstrated persistent resistance to AZM, whereas the heteroresistant isolate gradually reduced the number of bacteria from $10^{11} \mathrm{CFU}$ to $10^{5} \mathrm{CFU}$ when the AZM concentration was increased to $128 \mu \mathrm{g} / \mathrm{ml}$. This isolate was suspected as heteroresistant due to a more than eightfold difference between the lowest concentrations exhibiting maximum inhibition and the highest non-inhibitory concentration. Additionally, the AZM heterogeneous C. jejuni isolate genotype was compared with resistant C. jejuni, resistant C. coli, and an ATCC control strain (Table 1). At least one mutation was detected in all of the macrolide-resistant strains, but no mutation was observed in the ATCC 33560 control. Heterogeneous $C$. jejuni and resistant $C$. jejuni isolates possessed point mutations of 23S rRNA genes at positions 2075, whereas resistant $C$. coli presented the mutation at position 2230. The A2075G transitions were previously reported as efflux systems [14], and the number of gene copy mutations were proportional to the increased level of macrolide resistance. The two copies of this mutated gene conferred a lower macrolide MIC than

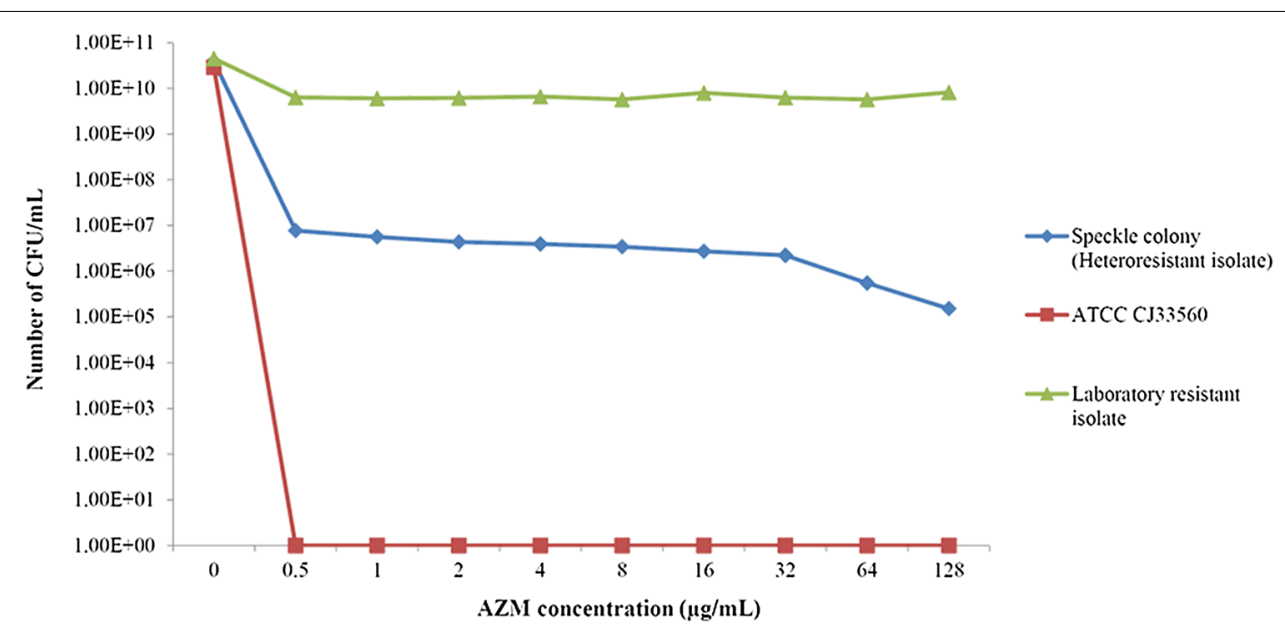

Fig. 2 The population analysis profiling (PAP) result of the laboratory resistant isolate, heteroresistant isolate, and ATCC 33560 control strain performed at the different AZM concentrations from 0 to $128 \mu \mathrm{g} / \mathrm{ml}$ by measuring the number of colony forming units (CFU)

Table 1 Determination of the minimal inhibitory concentration (MIC) and molecular characterization of the 23S rRNA gene mutations of heterogeneous C. jejuni, resistant C. jejuni, resistant C. coli, and C. jejuni ATCC 33560 against AZM

\begin{tabular}{|c|c|c|c|c|c|c|c|}
\hline \multirow[t]{2}{*}{ Organism } & \multirow[t]{2}{*}{ E-test to AZM } & \multicolumn{3}{|c|}{$\begin{array}{l}\text { 23S rRNA gene mutation at position } 2075 \\
\text { (A2075G) for C. jejuni }{ }^{b}\end{array}$} & \multicolumn{3}{|c|}{$\begin{array}{l}\text { 23S rRNA gene mutation at position } 2230 \\
\text { (A2230G) for C. colic }\end{array}$} \\
\hline & & Copy I (FI) & Copy II (FII) & Copy III (FIII) & Copy I (Fla) & Copy II (FIIa) & Copy III (FIIIa) \\
\hline C. colic & $\geq 256 \mu \mathrm{g} / \mathrm{ml}$ & & & & A2230G & A2230G & A2230G \\
\hline C. colic & $\geq 256 \mu \mathrm{g} / \mathrm{ml}$ & & & & A2230G & A2230G & A2230G \\
\hline C. colic & $\geq 256 \mu \mathrm{g} / \mathrm{ml}$ & & & & A2230G & A2230G & A2230G \\
\hline C. colic & $\geq 256 \mu \mathrm{g} / \mathrm{ml}$ & & & & A2230G & A2230G & A2230G \\
\hline C. jejunib & $\geq 256 \mu \mathrm{g} / \mathrm{ml}$ & A2075G & A2075G & A2075G & & & \\
\hline C. jejunib & $\geq 256 \mu \mathrm{g} / \mathrm{ml}$ & A2075G & A2075G & A2075G & & & \\
\hline C. jejunib & $\geq 256 \mu \mathrm{g} / \mathrm{ml}$ & A2075G & A2075G & A2075G & & & \\
\hline C. jejunib & $\geq 256 \mu \mathrm{g} / \mathrm{ml}$ & A2075G & A2075G & A2075G & & & \\
\hline C. jejuni (outer inhibition zone) ${ }^{\mathrm{a}}$ & $0.19 \mu \mathrm{g} / \mathrm{ml}$ & No mutation & No mutation & A2075G & & & \\
\hline C. jejuni (inner inhibition zone) ${ }^{\mathrm{a}}$ & $\geq 256 \mu \mathrm{g} / \mathrm{ml}$ & A2075G & No mutation & A2075G & & & \\
\hline ATCC33560 & Susceptible & No mutation & No mutation & No mutation & & & \\
\hline
\end{tabular}

\footnotetext{
a Heterogeneous C. jejuni
}

${ }^{\mathrm{b}}$ Resistant C. jejuni isolates a base substitution, $\mathrm{G}$ for A, were detected at position 2075 (corresponding to position 2059 in the nomenclature for E. coli [Genbank Accession No. J01695])

c Resistant C. coli isolates a base substitution, G for A, were detected at position 2230 (corresponding to position 2059 in the nomenclature or E. coli [Genbank Accession No. J01695], C. coli [Genbank Accession No. U09611]) 
the C. jejuni isolate with mutations in all three copies, which was previously mentioned regarding its relationship to in vivo selective pressure [7].

This heteroresistant C. jejuni demonstrated high MICs to macrolides like AZM and ERY, quinolones like NAL, and fluoroquinolones like CIP. Macrolides, generally considered the drug of choice for Campylobacter treatment [1], may be clinically less effective for heteroresistant infections. It should be noted that patient of this acute diarrheal illness was treated with $500 \mathrm{mg}$ of AZM for 3 days. Unfortunately, we were not able to obtain follow-up data regarding treatment results, so the effects of heteroresistance are not known. Nevertheless heteroresistant strains were previously reported that were responsible for treatment failure [15-18], which resulted in increased resistance to other antibiotics [19]. Due to the controversy of therapeutic implications of heteroresistance, relevant clinical data significance and additional experimental research is required [20]. Currently antimicrobial resistance in enteric pathogens is globally present. Despite this, the use of antimicrobial drugs in humans and animals are largely unrestricted [21]. The resistance to macrolides is more prevalent in Campylobacter isolates of animal origin, and the presence of macrolide-resistant isolates in the food chain increases the potential for human transmission [21]. Nonetheless, an increase of heteroresistant bacteria could be the result of high antibiotics use and possibly a driving mechanism for natural evolution to drug resistance [22] by proliferating and inhibiting susceptible subpopulations [20]. Hence, AST continues to play an important role in guiding therapy and epidemiological monitoring of resistance.

In conclusion, phenotypic and genotypic assessments of a C. jejuni isolate from a traveler to Nepal suffering acute diarrheal illness was confirmed as AZM heteroresistant. The conventional microbiology and phenotypic isolation techniques utilized for this study should be used to thoroughly examine atypical heteroresistant colonies. The original isolate, which presented as atypical colonies in the clear zone of inhibition, would suggest repetitive AST testing. The reproducible finding of atypical colonies in the clear zone of inhibition served as the primary observation of a heteroresistant strain, and the early detection of heteroresistant Campylobacter isolates enables medical providers to make appropriate treatment decisions. Regarding this knowledge gap, more research is needed to determine the true clinical impact of heteroresistance and its role in the development of fully-resistant bacterial populations. Proper laboratory and identification measures are needed to assess effective therapies and interventions for military members and civilian travelers suffering from persistent diarrhea.

\section{Authors' contributions}

$W L$ and SR participated in the conception and design of the study. WL, SR, and PW performed the laboratory work. SKS and PP were clinical coordinators, senior consultants, and fully supported this study in Nepal. WL and OS analyzed the data and wrote the manuscript. JC and LB contributed to the analysis and helped in writing the manuscript. All authors read and approved the final manuscript.

\section{Author details \\ ${ }^{1}$ Department of Bacterial and Parasitic Diseases, Armed Forces Research Institute of Medical Sciences, 315/6 Rajvithi Road, Bangkok 10400, Thailand. ${ }^{2}$ Walter Reed AFRIMS Research Unit, Kathmandu, Nepal. ${ }^{3}$ CIWEC Clinic Travel Medicine Center, Kathmandu, Nepal.}

\section{Acknowledgements}

We thank Carl J. Mason and Brett E. Swierczewski for supervision of this surveillance study. We also offer special thanks to (1) Brian P. Vesely, (2) Bacterial and Parasitic Diseases Department staff, AFRIMS, Thailand, (3) Walter Reed AFRIMS Research Unit staff, Kathmandu, Nepal and (4) CIWEC Clinic Travel Medicine Center staff, Kathmandu, Nepal for their assistance and kind support.

\section{Competing interests}

The authors declare that they have no competing interests.

\section{Authors' disclaimers}

Material has been reviewed by the Walter Reed Army Institute of Research. There is no objection to its presentation and/or publication. The opinions or assertions contained herein are the private views of the author, and are not to be construed as official, or as reflecting true views of the Department of the Army or the Department of Defense.

\section{Availability of data and materials}

Data sharing not applicable to this article.

\section{Consent for publication}

Not applicable.

\section{Ethics approval and consent to participate}

This study protocol was conducted in accordance with the ethical guidelines from the 'Code of Federal Regulations, Title 32, Part 219: Protection of Human Subjects' and was approved by Nepal Health Research Council Kathmandu, Nepal and Walter Reed Army Institute of Research (WRAIR), Silver Spring, MD, USA.

\section{Funding}

The study is supported by the Armed Forces Health Surveillance Branch (AFHSB) and it's GEIS (Global Emerging Infectious Disease Surveillance and Response) Section (Grant no. P0049_18_AF).

\section{Publisher's Note}

Springer Nature remains neutral with regard to jurisdictional claims in published maps and institutional affiliations.

Received: 12 March 2019 Accepted: 22 April 2019

Published online: 06 May 2019

\section{References}

1. Ge B, Wang F, Sjölund-Karlsson M, McDermott PF. Antimicrobial resistance in Campylobacter: susceptibility testing methods and resistance trends. J Microbiol Methods. 2013;95(1):57-67.

2. Pandey P, Bodhidatta L, Lewis M, Murphy H, Shlim DR, Cave W, et al. Travelers' diarrhea in Nepal: an update on the pathogens and antibiotic resistance. J Travel Med. 2011;18(2):102-8.

3. Center Centers for Diseases Control and Prevention (CDC). Incidence and trends of infection with pathogens transmitted commonly through foodfoodborne diseases active surveillance network, 10 U.S. sites, 1996-2012. MMWR Morb Mortal Wkly Rep. 2013;62:283-7. 
4. Blaser MJ, Engberg J. Clinical aspects of Campylobacter jejuni and Campylobacter coli infections. In: Nachamkin I, Szymanski CM, Blaser MJ, editors. Campylobacter. Washington, D. C.: ASM press; 2008. p. 99-121.

5. Alfredson DA, Korolik V. Antimicrobial resistance and resistance mechanisms in Campylobacter jejuni and Campylobacter coli. FEMS Microbiol Lett. 2007;277(2):123-32.

6. El-halfawy OM, Valvano MA. Antimicrobial heteroresistance: an emerging field in need of clarifies. Clin Microbiol Rev. 2015;28(1):191-207.

7. Ladely SR, Meinersmann RJ, Englen MD, Fedorka-Cray PJ, Harrison MA. 23S rRNA gene mutations contributing to macrolide resistance in Campylobacter jejuni and Campylobacter coli. Foodborne Pathog Dis. 2009;6(1):91-8.

8. Institute CLS. Methods for antimicrobial dilution and disk susceptibility testing of infrequently isolated or fastidious bacteria; approved guidelinesecond edition. CLSI Document M45-A2. 2010;30(18):2.

9. National Antimicrobial resistance Monitoring System. Retail Meat Report, Department of health and human services, USA. 2011. https://www.fda. gov/downloads/AnimalVeterinary/SafetyHealth/AntimicrobialResista nce/NationalAntimicrobialResistanceMonitoringSystem/UCM334834.pdf. Accessed 30 Jan 2019.

10. Poly F, Serichatalergs O, Schulman M, Ju J, Cates CN, Kanipes M, et al. Discrimination of major capsular types of Campylobacter jejuni by multiplex PCR. J Clin Microbiol. 2011;49:1750-7.

11. Meletis G, Tzampaz E, Sianou E, Tzavaras I, Sofianou D. Colistin heteroresistance in carbapenemase producing Klebsiella pneumoniae. J Antimicrob Chemother. 2011;66:946-7.

12. El-Halfawy OM, Valvano MA. Chemical communication of antibiotic resistance by highly resistant subpopulation of bacterial cells. PLoS ONE. 2013:8:e68874.

13. Ewer EC, Anisowicz SK, Ferguson TM, Seronello SE, Barnhill JC, Lustik MB, et al. Antibiotic resistance, molecular characterizations, and clinical manifestations of Campylobacteriosis at a military medical center in Hawail from 2012-2016: a retrospective analysis. Sci Rep. 2018;8(1):11736.
14. Cagliero C, Mouline C, Payot S, Cloeckaert A. Involvement of the CmeABC efflux pump in the macrolide resistance of Campylobacter coli. J Antimicrob Chemother. 2005;56(5):948-50.

15. Ariza J, Pujol M, Cabo J, Peña C, Fernández N, Liñares J, et al. Vancomycin in surgical infections due to methicillin-resistant Staphylococcus aureus with heterogeneous resistance to vancomycin. Lancet. 1999;353(9164):1587-8.

16. Wong S, Ho PL, Woo PC, Yuen KY. Bacteremia caused by staphylococci with inducible vancomycin heteroresistance. Clin Infect Dis. 1999;29(4):760-7

17. Mascellino MT, Oliva A, De Angelis M, Pontone S, Porowska B. Helicobac ter pylori infection: antibiotic resistance and eradication rate in patients with gastritis showing previous treatment failures. New Microbiol. 2018;41(4):306-9.

18. Kargarpour KM, Sadegh HR, Farmanfarmaei G, Masoumi M, Fateh A, Javadi $G$, et al. Evaluation of the impact of polyclonal infection and heteroresistance on treatment of tuberculosis patients. Sci Rep. 2018;7:41410. https://doi.org/10.1038/srep41410.

19. Sakoulas G, Alder J, Thauvin-Eliopoulos C, Moellering RC, Eliopoulos GM. Induction of daptomycin heterogeneous heterogeneous susceptibility in Staphylococcus aureus by exposure to vancomycin. Antimicrob Agents Chemother. 2006;50(4):1581-5.

20. Falagas ME, Makris GC, Dimopoulos G, Matthaiou DK. Heteroresistance: a concern of increasing clinical significance? Clin Microbiol Infect. 2008;14(2):101-4.

21. Mughini GL, Smid JH, Wagenaar JA, de Boer AG, Havelaar AH, Friesema $\mathrm{IH}$, et al. Risk factors for Campylobacteriosis of chicken, ruminant, and environmental origin: a combined case-control and source attribution analysis. PLOS ONE. 2012;7(8):e42599.

22. Morand B, Muhlemann K. Heteroresistance to penicillin in Streptococcus pneumonia. Proc Natl Acad Sci USA. 2007:104(35):14098-103.
Ready to submit your research? Choose BMC and benefit from:

- fast, convenient online submission

- thorough peer review by experienced researchers in your field

- rapid publication on acceptance

- support for research data, including large and complex data types

- gold Open Access which fosters wider collaboration and increased citations

- maximum visibility for your research: over $100 \mathrm{M}$ website views per year

At $\mathrm{BMC}$, research is always in progress.

Learn more biomedcentral.com/submissions 